\title{
Resting energy expenditure and optimal nutrition in critical care: how to guide our calorie prescriptions
}

\author{
Oren Zusman ${ }^{1,2^{*}}$ and Pierre Singer ${ }^{2,3}$
}

See related Letter by Berger et al. https://ccforum.biomedcentral.com/articles/10.1186/s13054-017-1612-6

We thank Berger et al. [1] for their interesting comment regarding our study [2]. Briefly, they suggest that "feeding progression days" might induce bias so that the administered calories/resting energy expenditure (REE) percentage (\% Adcal/REE) we show associated with better outcome is lower than the "true" mean. We agree that for the short-stayers included in the cited studies this remark is pertinent, but we would like to exclude studies based on predictive equations since they lack accuracy and may mislead our understanding. According to clinical practice [3], calories are usually increased progressively to the plateau target, but in addition, calories administered may vary during ICU stay due to interruptions in nutrition administration, making evaluations even more complicated.

Our study, using thousands of measured REEs acquired by indirect calorimetry, was performed from admission, with a local strategy to quickly increase calorie intake to target. Following Berger et al.'s suggestion, we present here a sensitivity analysis, including only feeding days from day 3 and onwards, based on the fact that, from this day, calorie intake didn't change significantly per day (Fig. 1 in our original study). We still found a significant association with mortality $(p=0.003)$. In addition, we have analyzed patients with more than ten evaluable ICU nutrition days. Figure 1 here shows the original curve along with the respective ones from the sensitivity analysis. The U-shaped curve is preserved, and the "optimal" point of \% Adcal/REE after excluding two days is similar ( $71 \%$ after excluding first two days, and $80 \%$ including only patients with more than ten evaluable nutrition days). So the first days' effect is minimal and does not change the study's message, surely not moving the target to $95-105 \%$ as proposed by Berger et al. This does not fit the results reported in a supplemental parenteral nutrition study [4], possibly because it was powered to demonstrate a reduction in morbidity and not in mortality.

Practically, daily calorie needs and administration cannot be expected to be constant. After reanalysis, our observation still stresses the importance of using $\%$ Adcal/REE as measured by indirect calorimetry, as it demonstrated association with reduced mortality. Our take home message remains that our aim should be to target $100 \%$ of REE and, due to practicalities of daily care, ultimately achieve $70-80 \%$ over the course of the ICU stay. This may serve as a strong basis for further studies.

\footnotetext{
*Correspondence: orenzu1@clalit.org.il; orenzusman@gmail.com

${ }^{1}$ Department of Internal Medicine E, Rabin Medical Center, Beilinson Hospital, Petah Tikva, Israel

${ }^{2}$ Department of General Intensive Care and Institute for Nutrition Research,

Rabin Medical Center, Beilinson Hospital, Petah Tikva, Israel

Full list of author information is available at the end of the article
} 


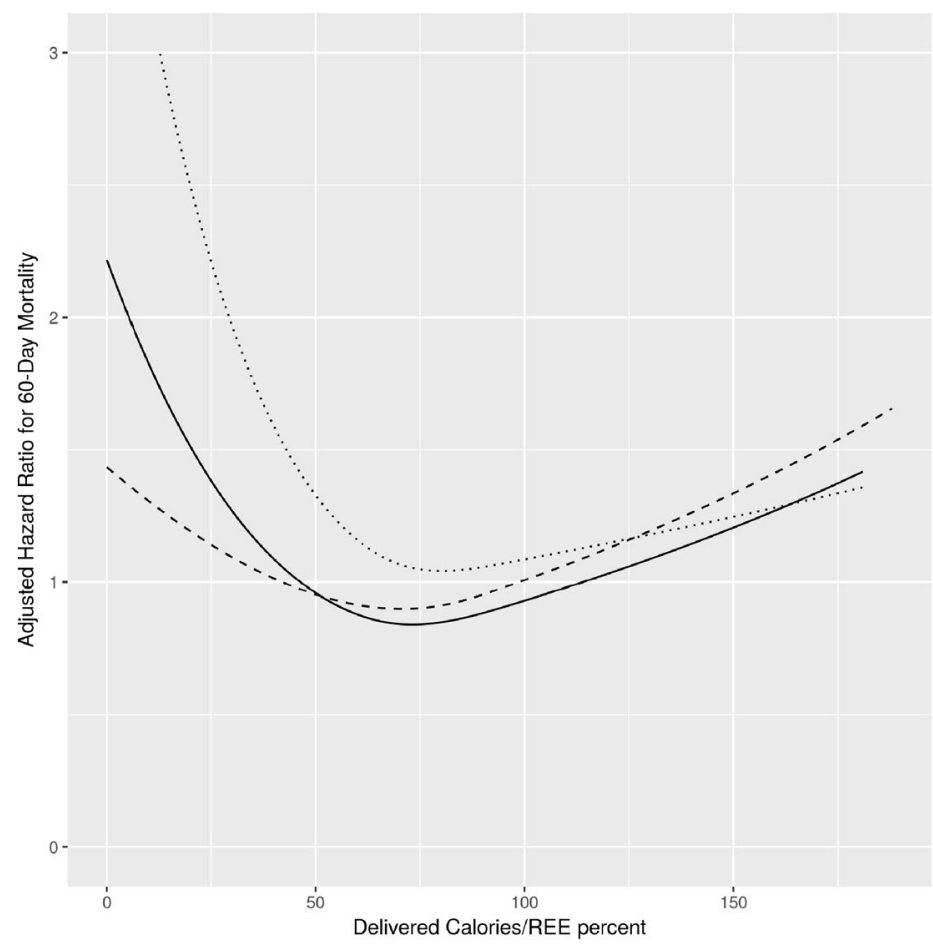

Fig. 1 Association of administered calories/REE percentage with mortality in different models

\section{Abbreviations}

$\%$ AdCal/REE: percentage administered calories/resting energy expeniture; ICU: intensive care unit

\section{Acknowledgements}

None.

\section{Funding}

Internal.

\section{Availability of data and materials}

The datasets generated during and/or analyzed during the current study are not publicly available due to privacy concerns and institutional policy.

\section{Authors' contributions}

OZ participated in the design of the study, performed the statistical analysis, and drafted the manuscript. PS conceived of and designed the study and participated in the analysis. Both authors took part in drafting the manuscript and approved the final form.

\section{Author information}

Not applicable.

\section{Competing interests}

The authors declare that they have no competing interests.

\section{Consent for publication}

Not applicable.

\section{Ethics approval and consent to participate}

The Rabin Medical Center institutional review board has authorized the original study and waived the need for informed consent due to the observational nature of the study, reference number 2017-0049.

\section{Publisher's Note}

Springer Nature remains neutral with regard to jurisdictional claims in published maps and institutional affiliations.

\section{Author details}

'Department of Internal Medicine E, Rabin Medical Center, Beilinson Hospital, Petah Tikva, Israel. ${ }^{2}$ Department of General Intensive Care and Institute for Nutrition Research, Rabin Medical Center, Beilinson Hospital, Petah Tikva, Israel. ${ }^{3}$ Sackler School of Medicine, Tel Aviv University, Tel Aviv, Israel.

Published online: 05 June 2017

\section{References}

1. Berger MM, Pichard C, Fontaine E. Optimal energy delivery and measured energy expenditure-impact of length of stay. Crit Care. 2017;21:39.

2. Zusman O, Theilla M, Cohen J, Kagan I, Bendavid I, Singer P. Resting energy expenditure, calorie and protein consumption in critically ill patients: a retrospective cohort study. Crit Care. 2016;20:367.

3. Bendavid I, Singer $P$, Theilla M, Themessl-Huber M, Sulz I, Mouhieddine M, Schuh C, Mora B, Hiesmayr M. NutritionDay ICU: A 7 year worldwide prevalence study of nutrition practice in intensive care. Clin Nutr. 2016. doi: 10.1016/j.clnu.2016.07.012. [Epub ahead of print]

4. Heidegger CP, Berger MM, Graf S, Zingg W, Darmon P, Costanza MC, et al. Optimisation of energy provision with supplemental parenteral nutrition in critically ill patients: a randomised controlled clinical trial. Lancet. 2013;381: 385-93. 